\title{
Particle accumulation in ureteric stents is governed by fluid dynamics: in- vitro study using a 'stent-on-chip' model
}

Ali Mosayyebi ${ }^{1,2}$, Qi Yann Yue ${ }^{1}$, Bhaskar K. Somani ${ }^{3}$, Xunli Zhang ${ }^{1,2}$, Costantino Manes ${ }^{4}$, Dario Carugo ${ }^{1,2, *}$

1. Mechanical Engineering Department, Faculty of Engineering and the Environment, University of Southampton, Southampton, UK

2. Institute for Life Sciences (IfLS), University of Southampton, Southampton, UK

3. Department of Urology, University Hospital Southampton NHS Trust, Southampton, UK

4. Department of Environment, Land and Infrastructure Engineering, Politecnico di Torino, Turin, Italy

Journal: $\quad$ Journal of Endourology

Type of Paper: $\quad$ Original Article

No. of Figs:

6

\section{*Correspondence to:}

\section{Dr Dario Carugo}

New Frontiers Fellow

Mechatronics and Bioengineering Science Research Groups

Institute for Life Sciences (IfLS)

Department of Mechanical Engineering

Faculty of Engineering and the Environment

University of Southampton

Highfield, Southampton SO17 1BJ

Office Tel: +44 (0) 2380593242

Keywords: Ureteric stent, ureter, microfluidic, encrustation, side holes, flow dynamics 


\section{Abstract}

\section{Objective}

To investigate the correlation between fluid dynamic processes and deposition of encrusting particles in ureteric stents.

\section{Materials and Methods}

Microfluidic models (referred to as 'stent-on-chip' or SOC) were developed to replicate relevant hydrodynamic regions of a stented ureter, including drainage holes and the cavity formed by a ureteric obstruction. Computational fluid dynamic (CFD) simulations were performed to determine the wall shear stress (WSS) field over the solid surfaces of the model, and the computational flow field was validated experimentally. Artificial urine was conveyed through the SOCs to measure the temporal evolution of encrustation via optical microscopy.

\section{Results}

It was revealed that drainage holes located well downstream of the obstruction had almost stagnant flow and low WSS (average $0.01 \mathrm{~Pa}$, at $1 \mathrm{~mL} / \mathrm{min}$ ), and thus suffered from higher encrustation rates. On the contrary, higher levels of WSS in holes proximal to the obstruction (average $\sim 0.04 \mathrm{~Pa}$, at $1 \mathrm{~mL} / \mathrm{min}$ ) resulted in lower encrustation rates in these regions. The cavity located nearby the obstruction was characterised by high levels of encrustation, due to the low WSS (average $1.6 \times 10^{-4} \mathrm{~Pa}$, at $1 \mathrm{~mL} / \mathrm{min}$ ) and the presence of flow vortices. Increasing the drainage flow rate from 1 to $10 \mathrm{~mL} / \mathrm{min}$ resulted in significantly lower deposition of encrusting crystals. 


\section{Conclusion}

The present study demonstrated an inverse correlation between deposition of encrusting bodies and the local WSS in a stented ureter model. Critical regions with low WSS and susceptible to encrustation were identified, including 'inactive' side holes (i.e., with minimal or absent flow exchange between stent and ureter) and the cavity formed by a ureteric occlusion. Findings from this study can open new avenues for improving the stent's design via fluid dynamic optimisation. 


\section{INTRODUCTION}

Ureteric stents are one of the most commonly used urological tools to provide urine drainage when this is impaired or blocked by obstructions, such as ureteral stones or tumors [1-3].

Since the introduction of the double-pigtail ureteric stent by Finney in 1978 [4], the stenting technology has undergone several developments [5]. However, stents still suffer from failures, and the associated morbidities and complications significantly affect a patient's quality of life and cause financial burden on national healthcare services [5]. Incidence of these failures depends on several factors including indwelling time, patients' fluid intake, urinary stasis for extrinsic compression, and access to healthcare. Encrustation and biofilm formation over the stent surface have been identified as primary determinants of stents' failure, with incidence rates of $58 \%$ and $28 \%$, respectively [6]. Whilst studies have been conducted to identify or develop materials and surface coatings inhibiting encrustation and bacterial adhesion, the dominant processes governing encrustation dynamics in ureteric stents are still not entirely clear [7, 8]. Among these, fluid dynamic processes have been largely overlooked although earlier theoretical studies have suggested that they may play a significant role on the initiation and growth of encrustation [9].

The present study attempts to fill this knowledge gap. It is herein hypothesised that encrustation rates are strongly correlated with a specific flow metrics called wall shear stress (WSS). In particular, it is argued that low and high levels of WSS are correlated with high and low encrustation rates, respectively. To test this hypothesis, 'stent-on-chip' (SOC) models were developed and employed in order to generate flow fields that resemble, in some measure, those encountered in a 'stented ureteric system'. Artificial urine was run into the SOCs and encrustation deposits (mainly in the form of crystals) were monitored over time. A computational fluid dynamic (CFD) model replicating the experiments was then implemented 
to compute WSS, so that it was possible to spatially correlate wall shear stress with the experimentally-observed encrustation maps, thus allowing the identification of regions prone to encrustation.

\section{MATERIALS AND METHODS}

\section{'Stent-on-chip' models: design rationale}

SOC models were designed to replicate relevant flow domains of a stented and occluded ureter, including:

(i) a large (1.5 mm wide) and a narrow ( $0.5 \mathrm{~mm}$ wide) channel, replicating the intra- and extraluminal compartments of a ureteric stent, respectively. Channels were separated by a $0.5 \mathrm{~mm}$ thick wall, mimicking the stent's wall.

(ii) An obsruction (or cavity), replicating an occlusion of the ureter lumen caused by either a ureteral stone (intrinsic obstruction) or a tumour (extrinsic obstruction). A proximal, complete occlusion of the ureter lumen was modelled (ureter diameter at the occlusion site $=2.5 \mathrm{~mm}$ ). Notably, earlier studies using full-scale models of a stented and occluded ureter observed typical features of cavity flow (including formation of laminar vortices), nearby a complete ureteric occlusion [10]. We will thus refer to this specific flow domain as 'cavity' or 'occluded cavity’ from herein.

(iii) Two drainage holes ( $0.8 \mathrm{~mm}$ wide), proximal (hole 1$)$ and distal (hole 2) to the obstruction.

The above dimensional characteristics of SOC models were defined to replicate those of commercial double-J stents (Cook® Medical, USA), as illustrated in Fig 1a,b,c. Two cylindrical chambers (5 $\mathrm{mm}$ in diameter) were also designed as inlet and outlet reservoirs in the SOCs. 
The total width and length of the microfluidic device were limited to $50 \mathrm{~mm}$ and $70 \mathrm{~mm}$, respectively (see Fig 2a), so that it could fit onto a microscope stage to monitor flow and encrustation processes in situ. Moreover, the length of the stent compartment at the inlet side of the device was defined to be greater than the entrance length $\left(L_{e}\right)$, allowing the flow to fully develop before reaching side hole $1 . L_{e}$ was determined from $0.06 \times \operatorname{Re} \times D_{h}$, where $D_{h}$ and $\operatorname{Re}$ correspond to the channel's hydraulic diameter and Reynolds number, respectively. The Reynolds number is defined as $\rho V D_{h} / \mu$, where $\rho, \mu$, and $V$ correspond to the fluid density, dynamic viscosity, and mean velocity, respectively. The mean velocity was determined from the ratio between the volumetric flow rate $(Q)$ and the inlet channel's cross-sectional area. Values of inlet volumetric flow rate investigated in the present study were in the range $0.1-$ $20 \mathrm{~mL} / \mathrm{min}$, following a previous study by Tofft et al. [11].

Although SOC models fail to make an exact replica of a stented ureter, they do capture relevant flow features, including a laminar vortex in the cavity formed by the obstruction and the lateral drainage through side holes (Fig 1b,c). Furthermore, they provide an hydrodynamically rich environment - particularly in terms of its WSS field - which includes both regions of low and high WSS. Thus they represent a useful investigational tool that, while maintaining the essential flow features encountered in stented ureteric systems, also allows for high-resolution mapping of encrustation rates at key hydrodynamic regions of interest.

\section{'Stent-on-chip' models: fabrication protocols}

SOC models were fabricated using a cost-effective technique described earlier, which combines micromilling with replica moulding [12]. The fabrication process comprised the following steps (Fig 2): (i) computationally assisted design (CAD) of the model geometry using SolidWorks (Dassault Systèmes Solidworks Corp., France) (Fig 2a). (ii) Generation of a negative mould by micromilling of the model architecture into a layer of poly(methyl 
methacrylate) (PMMA) (Fig 2b). (iii) Pouring of a liquid epoxy resin (yellow dual cartridge, RS Components Ltd., UK, at 1:1 weight ratio between components) on the negative mould. (iv) Curing of the epoxy layer at room temperature $\left(\sim 21^{\circ} \mathrm{C}\right.$, for $\left.\sim 100 \mathrm{~min}\right)$, and removal from the PMMA negative mould (Fig 2c). This layer contained a positive replica of the SOC architecture (positive mould). (v) Pouring of liquid poly(dimethylsiloxane) (PDMS, Sylgard® 184, Dow Corning Corporation, USA) over the positive mould. PDMS was produced at a curing agent:monomer ratio of 1:10 (by weight), degassed using a vacuum chamber, and then

cured overnight at ambient temperature. (vi) Removal of the cured PDMS layer from the positive mould, and bonding to a $1 \mathrm{~mm}$ thick glass layer (Corning® microscope slides, Sigma Aldrich, UK) by plasma treatment (Tepla300, Oxford Instrument, UK). A photograph of a finished stent-on-chip model is illustrated in Fig 2d.

\section{Simulation of the flow field}

CFD simulations were performed to characterise the flow field within SOC models. The simulated boundary conditions and fluid physical properties replicated the experimental ones. Simulations were performed at varying inlet volumetric flow rates, in the range $0.1-20$ $\mathrm{mL} / \mathrm{min}$. In designing the numerical mesh, particular attention was devoted to accurately resolve the wall shear stress distribution over the modelled solid surfaces. Additional details relating to the methods employed for model construction and numerical simulation are reported in supplementary section $\mathrm{S} 1$.

\section{Experimental characterisation of flow and encrustation processes}

In these experiments, the SOC model was placed horizontally over the microscope stage, to allow for optical access into the microfluidic channels.

Flow visualisation experiments were performed to qualitatively validate the simulated flow field. Fluorescent tracer beads (5.6 $\mu \mathrm{m}$ in diameter) were suspended in the fluid medium, and 
microscope images were acquired to reveal flow streamlines at selected locations within the SOC model. Further details about this method are reported in supplementary section S2.

Experiments were also conducted to quantify the formation of encrusting deposits. A supersaturated artificial urine (AU) was employed to investigate deposition and growth of encrusting crystals. The surrogate fluid was produced following a protocol designed by Keevil et al. [13], with minor modifications. Individual chemical constituents are reported in Table S1 (in supplementary section S3) and were purchased from Sigma Aldrich® (UK). The AU solution was prepared at $37^{\circ} \mathrm{C}$ and under continuous stirring; it had a $\mathrm{pH}$ of 6.8 , and was kept at $37^{\circ} \mathrm{C}$ during experimentation.

Experiments were performed at inlet flow rates of $1 \mathrm{~mL} / \mathrm{min}$ and $10 \mathrm{~mL} / \mathrm{min}[10,11,14]$, and five independent repeats were performed at each flow rate. In each experimental run, artificial urine was pumped into the microfluidic device for up to 90 minutes (see Fig 3), and microscope images were acquired at intervals of 15 minutes, using a CCD camera (exposure time $=1 \mathrm{~ms}$ ). Further details about the experimental set-up and protocols are reported in supplementary section S4. Image J (NIH, USA) was employed to process the microscope images and quantify the time evolution of encrustation, at selected locations within the device (see Fig 3). The image processing procotols are also described in supplementary section S4.

\section{Statistical Analysis}

A paired two sample t-test was performed to evaluate statistical differences between group means, using Microsoft Excel 2013 (Microsoft Corporation, USA). The significance level was set to 0.05 (i.e., differences were considered to be statistically significant if p-value $<0.05$ ). 


\section{RESULTS}

\section{Experimental validation of the simulations}

Fig 4a shows a comparison between the experimental and simulated (computational) flow streamlines, at three regions of interest within the model. All experimental repeats showed strong qualitative agreement with the numerical simulations in describing the flow field, in both proximal and distal side holes and the occluded cavity.

\section{WSS influences formation and growth of encrusting deposits in stents}

Simulations revealed the spatial distribution of WSS over the solid surfaces of the modelled SOCs. The contour of WSS over the bottom-wall is reported in Fig 4b,c. Hole 2 (i.e. located well downstream of the obstruction) represents a fairly stagnant flow region and is therefore characterised by low WSS (average WSS $=1.6 \times 10^{-4} \mathrm{~Pa}$ ). On the contrary, in hole 1 (i.e. right in proximity to the obstruction), the flow diversion caused by the obstruction imposes higher levels of WSS (average WSS $=0.038 \mathrm{~Pa}$ ). The occluded cavity, where the laminar vortex was observed, is also characterised by low levels of WSS (average WSS $=0.010 \mathrm{~Pa}$ ). It is therefore anticipated that regions potentially susceptible to the formation and growth of encrusting bodies include: side holes of the stent interested by negligible inter-compartmental flow exchange (i.e. hole 2) and the cavity formed by the obstruction.

This is confirmed in Fig 4c, which shows accumulation of encrusting deposits within side holes and the occluded cavity, at an inlet flow rate of $1 \mathrm{~mL} / \mathrm{min}$ (up to $90 \mathrm{~min}$ ). As anticipated, on the one hand, the side hole characterised by low WSS (hole 2) suffered from significant deposition of encrusting bodies. On the other hand, the side hole characterised by higher levels of WSS (hole 1) had a much lower degree of encrustation compared to hole 2. Notably, encrustation appeared to grow unevenly within a single side hole, with lower shear stress regions characterised by greater encrustation rates (see Fig 4c, for direct comparison between 
WSS and encrustation maps). The occluded cavity, characterised by low WSS, also experienced significant accumulation of encrusting bodies.

Fig 5 provides a more quantitative analysis of encrustation, as it shows its time evolution within the stent side holes and the occluded cavity, measured from the microscope images as $\%$ area covered by encrusting deposits. The area used for the calculation of the encrustation rate was $500 \mu \mathrm{m} \times 800 \mu \mathrm{m}$ (height $\times$ width), and is identified in Fig $4 \mathrm{c}$ by red boxes. Fig. 6a instead shows the mean \% encrusted area after 90 minutes at different regions of interest, and for both $1 \mathrm{~mL} / \mathrm{min}$ and $10 \mathrm{~mL} / \mathrm{min}$ inlet flow rates. Values are reported as average \pm standard deviation, and are compared for statistical significance.

At $1 \mathrm{~mL} / \mathrm{min}$, hole 1 had a lower coverage area compared to hole 2 (Fig 5a), which was characterised by lower WSS. The mean $\%$ coverage area after 90 min was equal to $7.6 \pm 0.8 \%$ (hole 1 ) and $13.8 \pm 3.8 \%$ (hole 2), respectively, and their difference was statistically significant (p-value $<0.05$, see Fig 6a). The growth rate of encrustation in hole $1\left(0.076 \mathrm{~min}^{-1}\right)$ was also significantly lower than the one in hole $2\left(0.143 \mathrm{~min}^{-1}\right)$. The cavity region was characterised by significantly high coverage area (Fig 5a); the mean value after 90 min was $26.9 \pm 6.0 \%$ and was statistically higher when compared to both side holes (p-values $<0.01$, see Fig. 6a). It should be noted that these values may depend on the positioning of the region of interest used for analysis (see Fig 4c), therefore encrustation rates in holes and cavity are not directly comparable.

Increasing the inlet flow rate up to $10 \mathrm{~mL} / \mathrm{min}$ resulted in a significant (up to 1-order of magnitude) decrease in the $\%$ coverage area (Fig $5 b$ ). The difference between mean $\%$ coverage area at $10 \mathrm{~mL} / \mathrm{min}$ and $1 \mathrm{~mL} / \mathrm{min}$ (after $90 \mathrm{~min}$ ) was statistically significant, for both side holes and the occluded cavity (p-values $<0.01$, see Fig. $6 a$ ). At $10 \mathrm{~mL} / \mathrm{min}$, the $\%$ coverage area in hole 1 and hole 2 (after $90 \mathrm{~min}$ ) was equal to $0.34 \pm 0.13 \%$ and $2.86 \pm 1.74 \%$, respectively, 
and their difference was not statistically significant ( $p$-value $>0.05$, see Fig. 6a). It was instead equal to $4.77 \pm 1.16 \%$ in the occluded cavity, which was statistically higher compared to both side holes (Fig 6a).

Figure $6 \mathrm{~b}$ shows the mean $\%$ coverage area (determined experimentally) vs. mean WSS (determined computationally), measured over the three regions of interest within SOCs, at both. 1 and $10 \mathrm{~mL} / \mathrm{min}$. Results demonstrate an inverse and non-linear correlation between size of encrusting deposits and WSS.

\section{DISCUSSION}

Ureteric stents are frequently used to restore drainage in the occluded ureter and form an essential tool for urological armamentarium. Despite their clinical success, stents suffer from complications and side effects. Formation and deposition of encrustation over the stent surface is widely recognised as a primary cause of complications, and is likely to be affected by the flow distribution in the stented ureter.

Few experimental models have been developed and employed to investigate flow processes associated with ureteric stents, either in-vitro or ex-vivo [15-20]. Using an artificial dynamic model, Gorman and co-authors measured lower levels of encrustation in stents exposed to dynamic flow conditions as opposed to static conditions, suggesting a significant "cleaning" action of the bulk flow [17]. Moreover, some studies revealed that side holes of the stent are often associated with higher deposition of encrustation, and suggested that holes may act as initial anchoring sites for encrustation [20,21]. This is also confirmed to some extent by the inspection of stents retreived from patients, where it was observed that the majority of side holes were plugged with crystals [22]. It is also well known that proximal and distal coils of a ureteric stent often suffer from encrustation, as reported in clinical studies [23, 24]. This is 
likely due to the stagnant nature of the flow in those regions, resulting in low WSS levels over the inner and outer surfaces of the coil.

Moreover, Clavica et al. [10] argued that laminar vortices forming in the vicinity of obstructions may also be considered as encrustation "hot spots", as they are are effective in entrapping particles suspended in the fluid and therefore in promoting their deposition.

Waters et al. $[9,25]$ employed a theoretical model to study the flow field in a stented ureter and discussed its relation to stent encrustation. They concluded that differences in the crystal structure, chemical composition and localisation of encrustations could depend strongly on the local urine environment.

The studies cited above suggest that, despite the very low-flow regime (i.e. akin to almost static conditions) normally encountered in stented ureteric systems, flow processes may dictate where, and at which rate, encrustations can grow over the stent surface. This is extremely relevant because, if confirmed, it can open new avenues for improving the stent's design via fluid dynamic optimisation. For instance, earlier computational studies have shown that increasing the number of side holes could increase the drainage flow rate [26], although this effect was minimal in the absence of a ureteric occlusion. In a prospective randomized clinical study by Damiano et al. [27], no differences in quality of life, urinary symptoms, and pain were found when using stents with different diameter, suggesting that further efforts should be devoted to more effectively improve the flow dynamic performance of stents.

Unfortunately, the literature is inconclusive on this specific issue as it lacks of systematic studies addressing the direct correlation between flow metrics and encrustation rates. The microfluidic-based models of a stented ureter developed in this study allowed to reveal, for the first time, a robust inverse correlation between deposition of encrusting bodies and the local WSS in a stented ureter model. 
Importantly, the flow features in the SOC models were in agreement with previous studies where more accurate replicas of stented ureters were employed [10, 28]. In the presence of a ureteric obstruction, inter-compartmental fluid exchange occurred only in the first side hole post-obstruction, coherently with earlier numerical studies [28], and a laminar vortex formed within the obstructed cavity as previously observed by Clavica et al. using a full-scale artificial ureter model [10].

Moeover, we identified critical regions susceptible to encrustation, which included 'inactive' side holes (i.e., with minimal or absent flow exchange between stent and ureter) and the cavity formed by a ureteric occlusion. In the cavity, encrustation occurred due to the generally low WSS levels and perhaps the trapping effect caused by the laminar vortex. Increasing WSS resulted in a significant reduction in particle deposition rates in all regions of the model investigated. Increased WSS levels in these critical regions could be achieved by altering the architecture of the stent locally, to promote fluid drainage through side holes.

In a full-scale stent, there are several factors than can potentially affect the flow and wall shear stress distribution over a stent, including stent's thickness and inner diameter, and the number, size and shape of its side holes. These parameters will be investigated in the future, using both SOC and full-scale numerical models of the stent. The effect of patient's posture (i.e. orientation of the stent) on crystals' deposition will also be studied.

Moreover, stent encrustation is a complex process involving several physical, chemical and micro-biological factors, each acting over different temporal scales. In this study, we focused on the deposition of encrusting crystals - a process that is likely to play an important role in stent encrustation, given the low-flow regime encountered in a stented ureter. Future work will however investigate the effect of changing the fluid chemical composition and $\mathrm{pH}$ on the formation and growth of crystals. For this purpose, microfluidic devices could be integrated 
with micro-Computed Tomography or Raman scattering technologies to investigate crystals structure and chemical composition in situ.

Despite they replicate relevant features of the flow field in a stented ureter, SOC models do not mimic some characteristic behaviours of a physiological ureter, including distensibility of the ureteral wall, ureteral peristalsis, and ability to adapt to pyelic pressures in case of obstruction. Moreover, whilst different levels of ureteric occlusion may occur in-vivo (encompassing total and partial obstruction), in this study we only replicated a complete occlusion of the lumen.

\section{CONCLUSIONS}

In the present study we developed microfluidic-based models of the stented and occluded ureter, and demonstrated an inverse correlation between wall shear stress and deposition of encrusting particles. Regions susceptible to encrustation were identified, which included 'inactive' side holes and the cavity formed by a complete occlusion of the ureter lumen.

As a result of our study, it could be speculated that the majority of side holes in a real stented ureter system can act as stagnation sites, and therefore represent potential "hot spots" for the anchoring and deposition of encrusting deposits. These hydrodynamic regions can then provide an enviroment for the growth of larger crystals and the entrapment of bacteria to form a complex bio-chemical layer, as described in previous studies [29].

In conclusion, the microfluidic models developed in this study provide a fast, cost-effective (in terms of fabrication costs), and reliable screening platform for investigating the interplay between flow processes and encrustation dynamics in stents. 


\section{ACKOWLEDGMENTS}

Authors would like to thank the Faculty of Engineering and the Enviroment (FEE) at the University of Southampton, $\mu$-VIS X-Ray Imaging Centre at Southampton, and the EPSRCfunded NAMRIP (Network on Antimicrobial Resistance and Infection Prevention, project reference: EP/M027260/1) for having financially supported the research outlined in this manuscript.

\section{AUTHOR DISCLOSURE STATEMENT}

No competing financial interests exist.

\section{REFERENCES}

1. Abrams, H.L., Abrams' angiography: interventional radiology. 2006, Lippincott Williams \& Wilkins. p. 669 - 697.

2. Kulkarni, R., Ureteric Stenting. 2017, John Wiley \& Sons. p. 1-55.

3. Chung, S.Y., et al., 15-year experience with the management of extrinsic ureteral obstruction with indwelling ureteral stents. The Journal of urology, 2004. 172(2): p. $592-595$.

4. Finney, R.P., Experience with New Double J-Ureteral Catheter Stent. Journal of Urology, 1978. 120(6): p. 678-681.

5. Lange, D., et al., Ureteral stent-associated complications - where we are and where we are going. Nature Reviews Urology, 2015. 12(1): p. 17-25.

6. Keane, P.F., et al., Characterization of biofilm and encrustation on ureteral stents in vivo. British Journal of Urology, 1994. 73(6): p. 687-691.

7. Mosayyebi, A., et al., Engineering solutions to ureteral stents: material, coating and design. Central European journal of urology, 2017. 70(3): p. 270. 
8. Lange, D. and B.H. Chew, Update on ureteral stent technology. Therapeutic Advances in Urology, 2009. 1(3): p. 143-148.

9. Waters, S.L., et al., Ureteric stents: investigating flow and encrustation. Proceedings of the Institution of Mechanical Engineers Part H-Journal of Engineering in Medicine, 2008. 222(H4): p. 551-561.

10. Clavica, F., et al., Investigating the flow dynamics in the obstructed and stented ureter by means of a biomimetic artificial model. PloS one, 2014. 9(2): p. e87433.

11. Tofft, H.P., et al., Renal Pelvic Peristalsis in Pigs during Standardized Flow Rate Variations. Urologia internationalis, 1986. 41(4): p. 292-298.

12. Carugo, D., et al., Facile and cost-effective production of microscale PDMS architectures using a combined micromilling-replica moulding ( $\mu M i$-REM) technique. Biomedical microdevices, 2016. 18(1): p. 4.

13. Brooks, T. and C.W. Keevil, A simple artificial urine for the growth of urinary pathogens. Lett Appl Microbiol, 1997. 24(3): p. 203-6.

14. Hobbs, T., et al., Evaluation of Biofilm Induced Urinary Infection Stone Formation in a Novel Laboratory Model System. The Journal of urology, 2018. 199(1): p. 178-185.

15. Olweny, E.O., et al., Flow characteristics of 3 unique ureteral stents: investigation of a poiseuille flow pattern. The Journal of urology, 2000. 164(6): p. 2099-2103.

16. Stoller, M., et al., An in vitro assessment of the flow characteristics of spiral-ridged and smooth-walled JJ ureteric stents. BJU international, 2000. 85(6): p. 628-631.

17. Gorman, S.P., et al., Design and validation of a dynamic flow model simulating encrustation of biomaterials in the urinary tract. Journal of pharmacy and pharmacology, 2003. 55(4): p. 461-468.

18. Clavica, F., et al., Investigating the Flow Dynamics in the Obstructed and Stented Ureter by Means of a Biomimetic Artificial Model. Plos One, 2014. 9(2). 
19. Choong, S., S. Wood, and H. Whitfield, A model to quantify encrustation on ureteric stents, urethral catheters and polymers intended for urological use. BJU international, 2000. 86(4): p. 414-421.

20. Gorman, S.P., et al., Mechanical performance of polyurethane ureteral stents in vitro and ex vivo. Biomaterials, 1997. 18(20): p. 1379-1383.

21. Gordon, N., Stalactites and Stalagmites: Crystal Formation on Ureteric Double-J Stents. BJU International, 1989. 64(2): p. 195-197.

22. Buchholz, N., et al., Handbook of Urinary Stents: Basic Science and Clinical Applications. 2016, JP Medical Ltd. p. 7-14.

23. Kawahara, T., et al., Ureteroscopic removal of forgotten ureteral stent. BMJ case reports, 2012. 2012: p. bcr0220125736.

24. Singh, I., et al., Severely encrusted polyurethane ureteral stents: management and analysis of potential risk factors. Urology, 2001. 58(4): p. 526-531.

25. Siggers, J.H., et al., Flow dynamics in a stented ureter. Mathematical medicine and biology: a journal of the IMA, 2008. 26(1): p. 1-24.

26. Kim, K.-W., et al., Numerical analysis of the effect of side holes of a double J stent on flow rate and pattern. Bio-medical materials and engineering, 2015. 26(s1): p. S319S327.

27. Damiano, R., et al., Does the size of ureteral stent impact urinary symptoms and quality of life? A prospective randomized study. European urology, 2005. 48(4): p. 673-678.

28. Tong, J.C., E.M. Sparrow, and J.P. Abraham, Numerical simulation of the urine flow in a stented ureter. Journal of biomechanical engineering, 2007. 129(2): p. 187-192.

29. Wilks, S.A., M.J. Fader, and C.W. Keevil, Novel Insights into the Proteus mirabilis Crystalline Biofilm Using Real-Time Imaging. Plos One, 2015. 10(10). 


\section{Figure legends}

Fig 1 (a) Two-dimensional (2D) schematic of the urinary system, with a simplified representation of extrinsic (e.g. tumour) or intrinsic (e.g. stone) causes of ureteric obstruction. (b) Schematic representation of key hydrodynamic regions within a stented ureter, located in the vicinity of a ureteric obstruction. These include two drainage holes (hole 1 and 2), the intraand extra-luminal compartments of the stent, and the cavity formed by a complete obstruction of the ureter lumen (this is also highlighted in red in (a)). Stent-on-chip models were designed to replicate these hydrodynamic regions. (c) Key geometrical properties of SOC models. Dimensions were taken from a commercial double-J stent (Cook® Medical, USA), which has an extended length of $40 \mathrm{~cm}$, inner diameter of $1.5 \mathrm{~mm}$, wall thickness of $0.5 \mathrm{~mm}$, and side hole diameter of $0.8 \mathrm{~mm}$.

Fig 2 Combined micromilling-replica moulding process employed to fabricate SOC models. Images show (a) a CAD design of the device, (b) the micromilled, PMMA-based negative mould (with a zoomed-in view of the side holes), (c) the epoxy-based positive mould casted from the micromilled mould, and (d) the PDMS channel architecture bonded to a glass slide, upon treatment with oxygen plasma.

Fig 3 (a) Experimental setup employed for visualising encrustation in-situ. It comprised a reservoir containing artificial urine (AU), a peristaltic pump (Minipuls3, Gilson Inc., France), the SOC device placed on a inverted microscope stage (Wilovert 30, Helmut Hund GmbH, Germany), and a CCD camera (GXCAM-HICHROME SII, GT-Vision Ltd., UK) connected to a PC for image acquisition. (b) Flowchart outlining the image processing steps performed to 
quantify encrustation within selected regions of the SOC model. ImageJ software (NIH, USA) was employed for image processing.

Fig 4 (a) Experimental fluid streamlines obtained from flow visualisation tests, and corresponding streamlines determined from CFD simulations. Results were taken at a fixed volumetric flow rate of $1 \mathrm{~mL} / \mathrm{min}$, and arrows indicate the flow direction. (b) Contour of WSS magnitude at the bottom plane (bed) of the SOC model, computed numerically. Blue and red colours correspond to low and high levels of WSS, as indicated in the coloured scale bar. (c) Contours of WSS at three key hydrodynamic regions within the model (hole 1, hole 2, and the cavity) and the corresponding microscope images showing encrusting deposits, at an inlet flow rate of $1 \mathrm{~mL} / \mathrm{min}$. Images were taken at $0 \mathrm{~min}$ and $90 \mathrm{~min}$ from the beginning of the experiment $(n=5)$. Red boxes correspond to the regions of interest, over which the \% coverage area occupied by encrustation was calculated. Please refer to the scale bar in (b) for WSS values.

Fig 5 Time evolution of the $\%$ area covered by encrustation, at specific regions of interest within the SOC models (side holes and cavity). Results are reported for inlet flow rate of (a) 1 $\mathrm{mL} / \mathrm{min}$ and (b) $10 \mathrm{~mL} / \mathrm{min}$, and are expressed as the mean \pm standard deviation of five independent repeats.

Fig 6 (a) Mean \% coverage area after $90 \mathrm{~min}$, at both $1 \mathrm{~mL} / \mathrm{min}$ (left) and $10 \mathrm{~mL} / \mathrm{min}$ (right), measured in hole 1 , hole 2, and the occluded cavity. Statistically significant differences between mean values are indicated with one asterisk (for $0.01<\mathrm{p}$-value $<0.05$ ) or two asterisks (for p-value $<0.01$ ). (b) Mean \% coverage area plotted as a function of mean WSS, at both 1 and $10 \mathrm{~mL} / \mathrm{min}$. Values were meased in hole 1 , hole 2 , and the occluded cavity. 


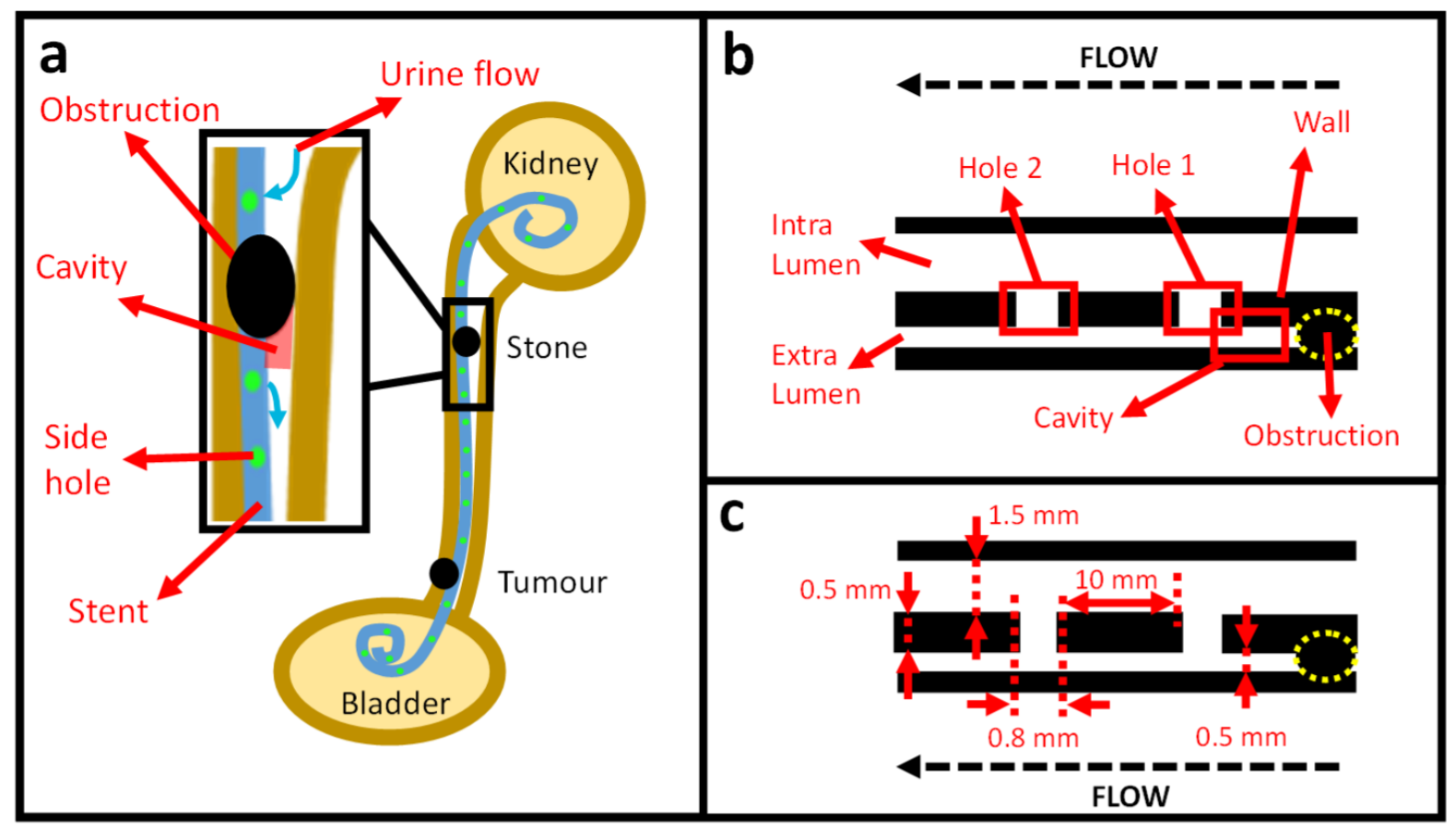

Figure 1

Side holes

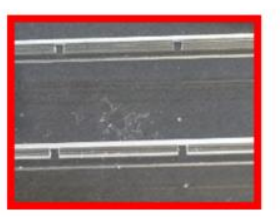

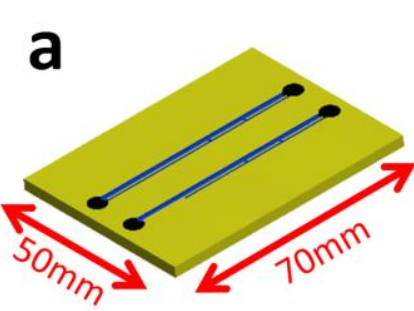

CAD design

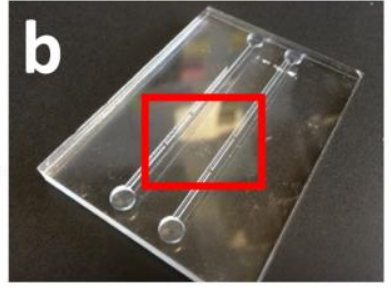

Milled mould

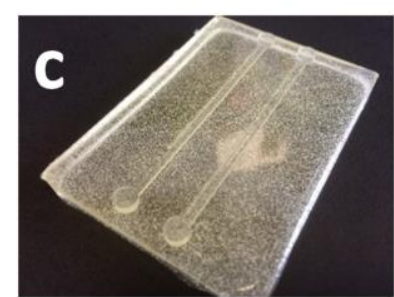

Epoxy mould

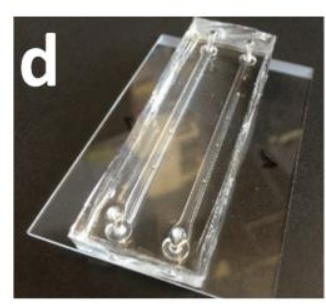

Sealed chip

Figure 2 


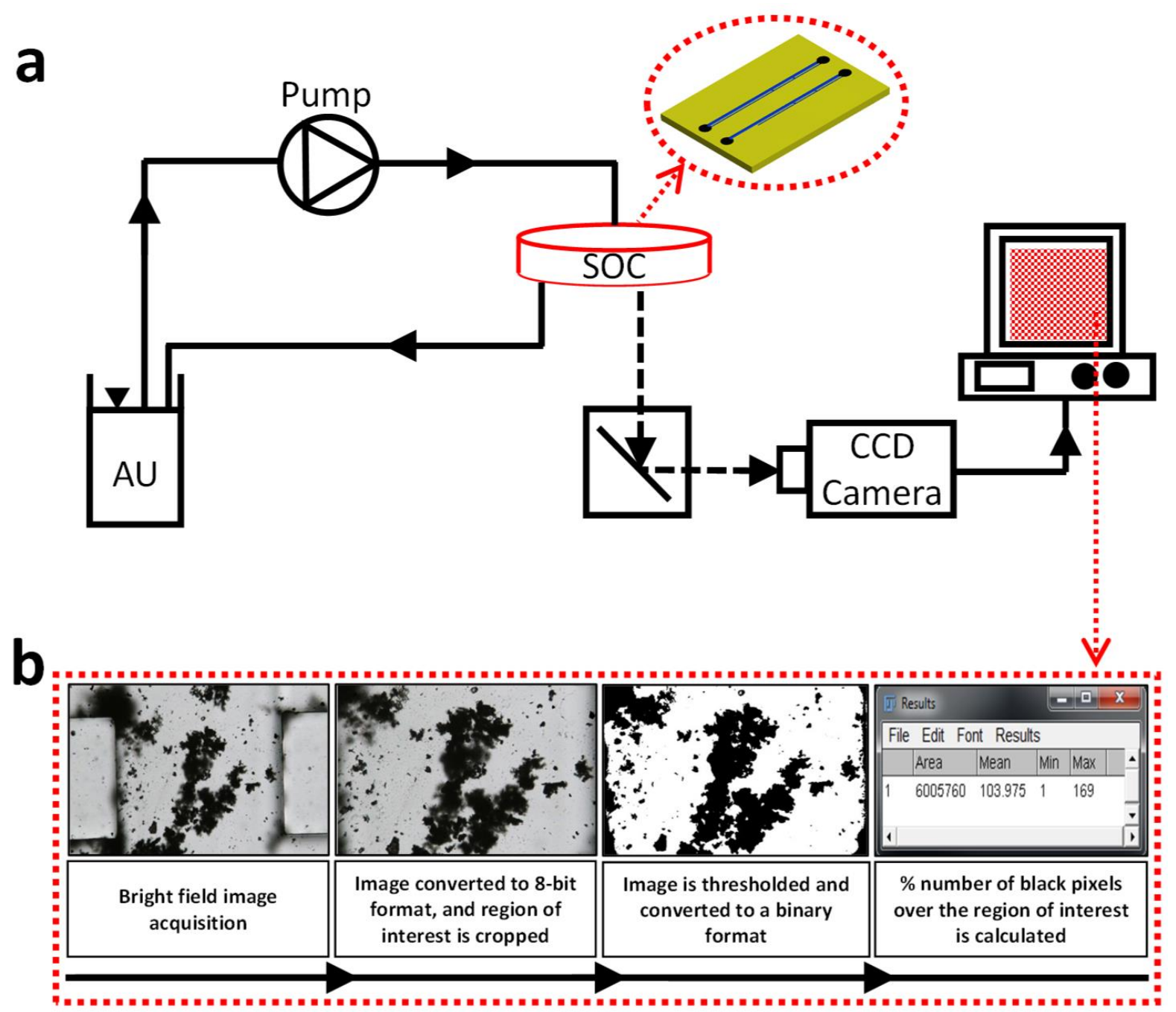

Figure 3 
a
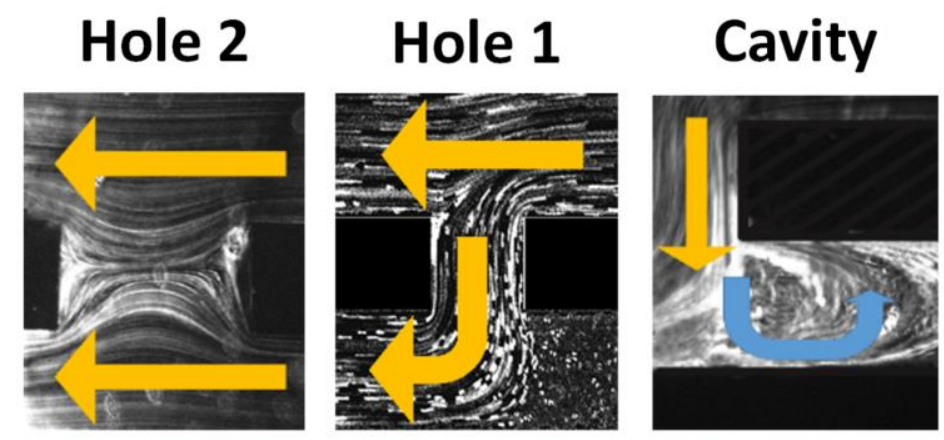

Experimental
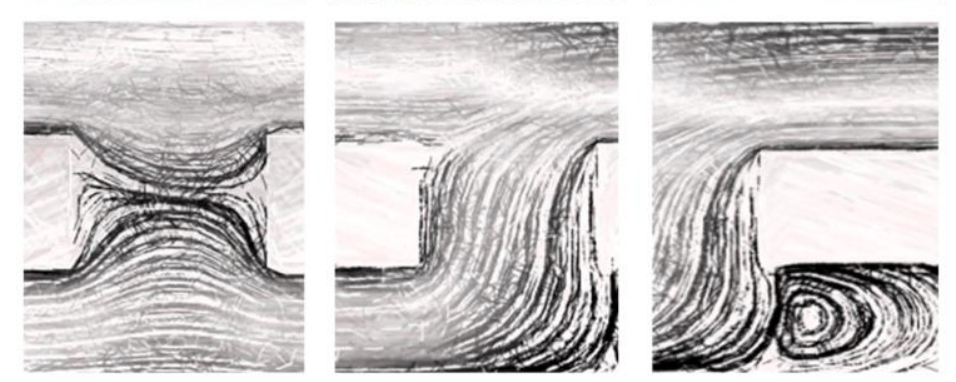

Computational

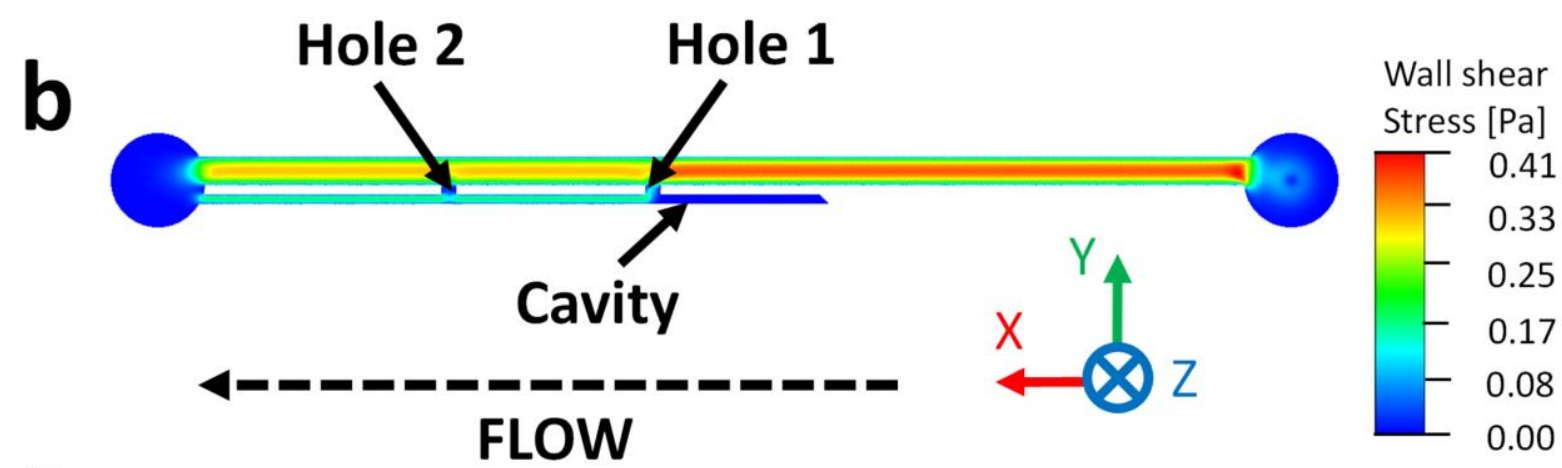

C
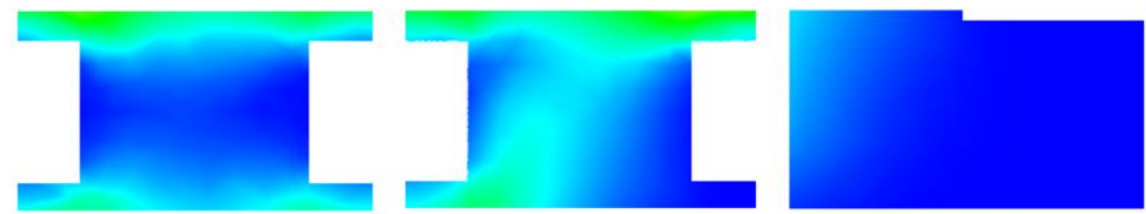

Wall shear

(simulation)
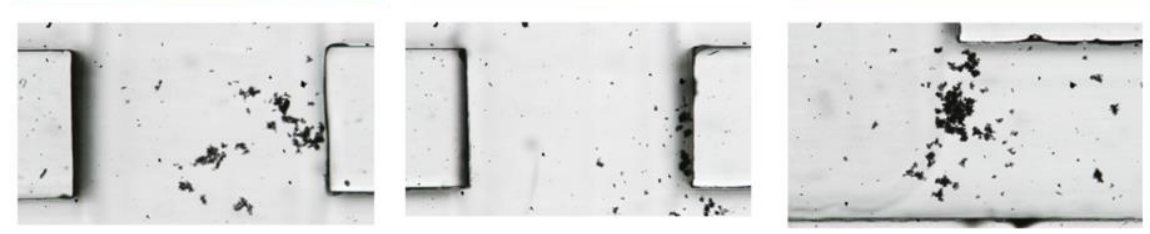

Start

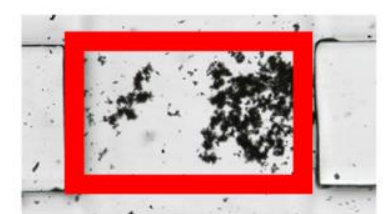

Hole 2

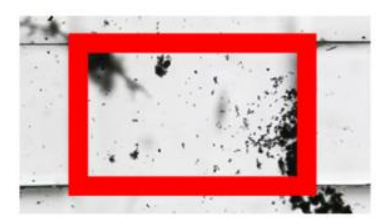

Hole 1

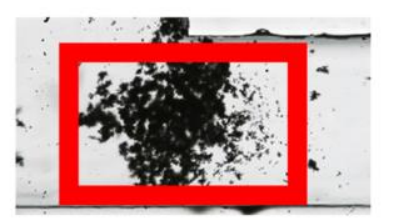

Cavity

Figure 4 
a

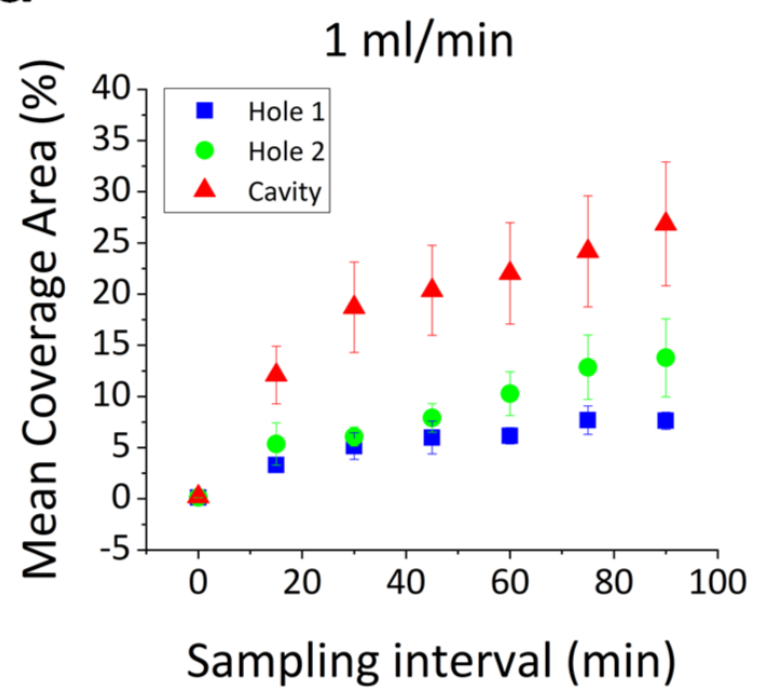

b

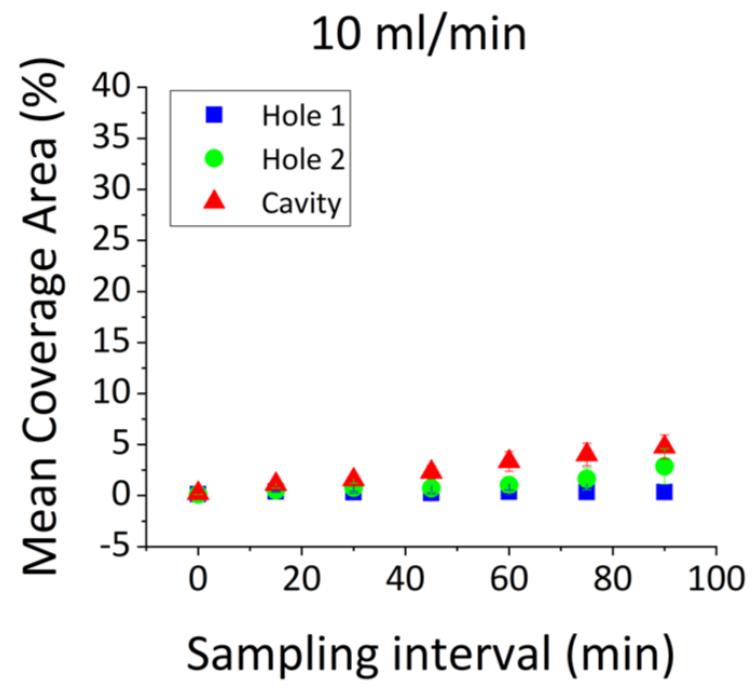

Figure 5 

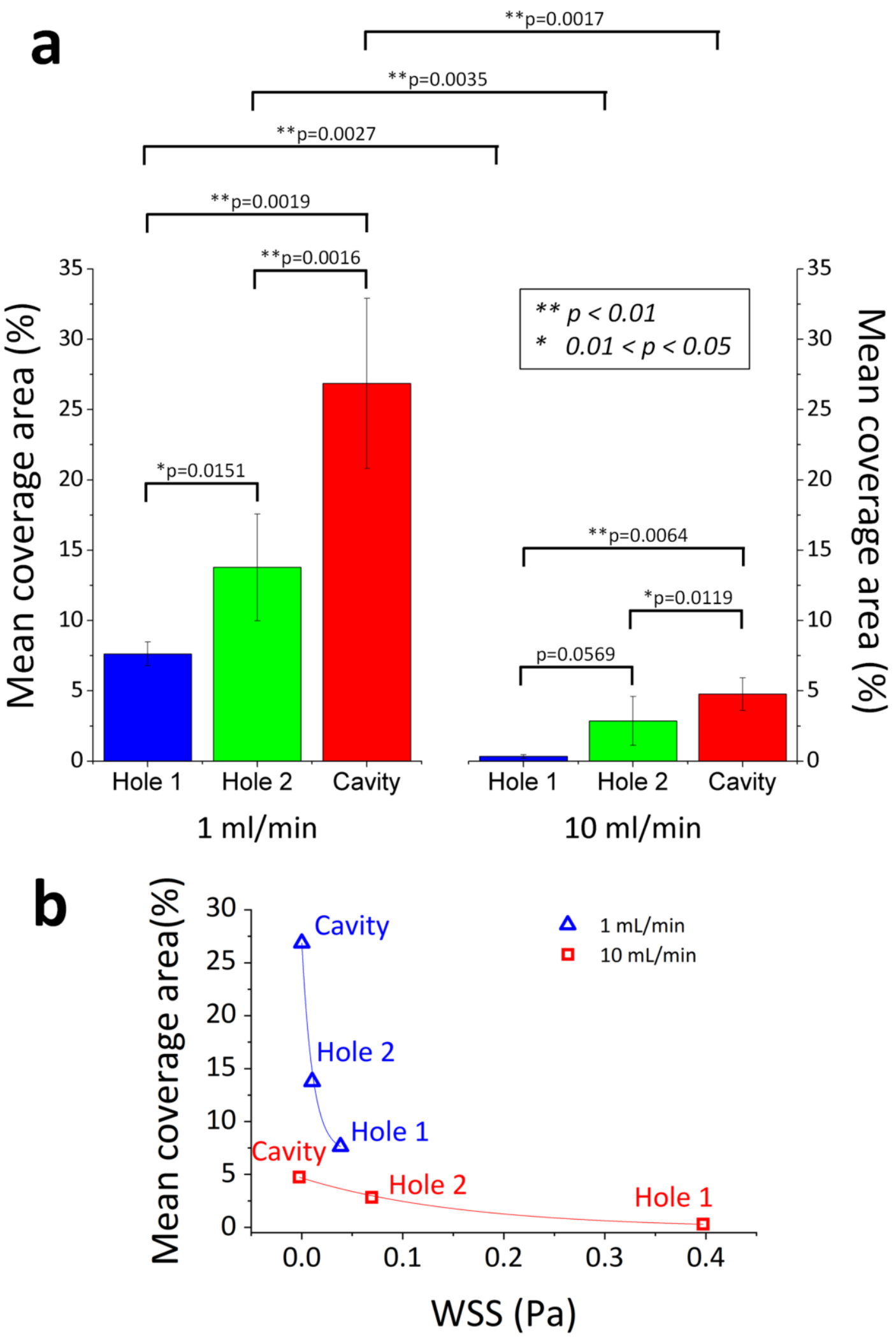

Figure 6 


\section{SUPPLEMENTARY INFORMATION}

\section{S1. Computational Fluid Dynamic (CFD) simulation methods}

Computational fluid dynamics (CFD) simulations were performed to characterise the flow field within SOC models. In this method, the governing equations describing the interaction of liquids with physical boundaries are solved computationally. In a first step, the device geometry was constructed using ICEM CFD 17.0 (Ansys Inc., USA). The volume occupied by the fluid was then discretised in finite volumes of tetrahedral shape. The number of mesh volumes $(17,770,735)$ and their size $(0.1 \mathrm{~mm})$ were defined subject to a compromise between solution robustness and computational cost. In other words, the $0.1 \mathrm{~mm}$ size was defined after carrying out a mesh dependence study. Ansys ${ }^{\circledR}$ Fluent 17.0 (Ansys Inc., USA) was subsequently employed to define the relevant physical models and boundary conditions, and to solve for mass and momentum conservation equations. A mass flow boundary condition was set at the model inlet, while atmospheric pressure was imposed at the outlet. A no-slip boundary condition was imposed at the ureter and stent walls.

A steady state, laminar flow model was employed to solve for the governing equations, given that the inlet Reynolds number was estimated to vary from 1.6 to $\sim 330$. Urine (which has comparable density and dynamic viscosity to water [1]) was assumed to be a Newtonian and incompressible fluid, with density of $997.044 \mathrm{~kg} / \mathrm{m}^{3}$ and dynamic viscosity of $0.001 \mathrm{~Pa} \cdot \mathrm{s}$.

Simulations were performed at varying inlet flow rates. Particular attention was devoted to the fluid velocity magnitude $(|\boldsymbol{v}|)$ and the wall shear stress (WSS), as potential determinants of the formation and growth of encrusting deposits. These were defined as follows:

$$
|v|=\sqrt{\left|v_{x}\right|^{2}+\left|v_{y}\right|^{2}+\left|v_{z}\right|^{2}}
$$


where $v_{x}, v_{y}$, and $v_{z}$ represent the components of the velocity vector in the $x$-, $y$ - and $z$-direction, respectively. And

$$
\boldsymbol{W S S}=-\mu \frac{\partial v}{\partial n}
$$

where $\boldsymbol{n}$ is the unit vector normal to the wall.

\section{S2. Flow visualisation experiments}

Flow visualisation experiments were performed to visualise the flow streamlines within the SOC models, and compare them with those calculated numerically. The experimental setup comprised a syringe pump (Harvard ®, apparatus pump33) loaded with a $50 \mathrm{~mL}$ plastic syringe (BD® Plastic). Polytetrafluoroethylene (PTFE) tubing (inner diameter: 1/32" and outer diameter: 1/16", Cole-Palmer $\left.{ }^{\circledR}, \mathrm{UK}\right)$ connected the syringe to the microfluidic device inlet, and the device outlet to a reservoir. The microfluidic device was placed on the stage of a fluorescent microscope equipped with laser illumination (Nikon ${ }^{\circledR}$, USA), for visualisation of fluorescent flow tracers. Tracers were $5.6 \mu \mathrm{m}$ diameter polystyrene beads (at a concentration of $2 \times 10^{4}$ beads $/ \mathrm{mL}$ ) loaded with a fluorescent dye excitable at $633 \mathrm{~nm}$ and emitting at $680 \mathrm{~nm}$. Images were acquired using a CCD camera (ORCA-R2, Hamamatsu Photonics K.K., Japan), with an exposure time of $10 \mathrm{sec}$ to reveal the flow streamlines. Experiments were performed at a fixed flow rate of $1 \mathrm{~mL} / \mathrm{min}$.

\section{S3. Artificial Urine Composition}


Table 1 List of chemicals used for producing the artificial urine (AU), including corresponding molecular weight and concentration in the final AU solution. The chemical composition of the urine surrogate was taken from Keevil et al. [2], with minor modifications.

\begin{tabular}{l|c|c}
\hline Component & $\begin{array}{c}\text { Molecular } \\
\text { Weight }\end{array}$ & Concentration (mmol/L) \\
\hline \hline Lactic acid & 90.08 & 1.1 \\
\hline Citric acid & 192.12 & 2 \\
\hline Sodium bicarbonate & 84.01 & 25 \\
\hline Urea & 60.06 & 170 \\
\hline Calcium chloride dihydrate & 147.00 & 2.5 \\
\hline Sodium chloride & 58.44 & 2 \\
\hline Magnesium sulphate heptahydrate & 246.47 & 10 \\
\hline Sodium sulphate decahydrate & 142.05 & 7 \\
\hline Potassium dihydrogen phosphate & 136.09 & 25 \\
\hline Ammonium chloride & 53.49 & 2 \\
\hline
\end{tabular}

\section{S4. Experimental quantification of encrustation}

The experimental setup employed to quantify accumulation of encrusting deposits within SOCs comprised a reservoir containing artificial urine, a peristaltic pump (MINIPULS3, Gilson, Inc., France), and the microfluidic device placed horizontally on the stage of an inverted optical microscope (Wilovert 30, Helmut Hund GmbH, Germany). Tubing (PTFE, Cole-Parmer®) connected the device to both the reservoir and the pump. A CCD camera (GXCAMHICHROME SII, GT-Vision Ltd., UK) was employed to acquire bright field microscope images of encrustation, at exposure time of $1 \mathrm{~ms}$.

ImageJ software (NIH, USA) was employed to process the images and quantify the time evolution of encrustation at selected locations within the devices. The processing steps were as follows: (i) bright field images were converted to 8-bit format, and areas of interest within the image were manually selected (cropped). (ii) Images were thresholded in to a black-and-white binary image format, where values equal to 255 corresponded to encrusting deposits; and (iii) the percentage area occupied by encrustation was quantified. 


\section{References}

1. Tong, J.C.K., E.M. Sparrow, and J.P. Abraham, Numerical Simulation of the Urine

Flow in a Stented Ureter. Journal of Biomechanical Engineering, 2006. 129(2): p. 187192.

2 .

Brooks, T. and C.W. Keevil, A simple artificial urine for the growth of urinary pathogens. Lett Appl Microbiol, 1997. 24(3): p. 203-6. 